\title{
Estimation spatialisée
} de l'évapotranspiration des cultures irriguées par télédétection : application à la gestion de l'irrigation dans la plaine du Haouz (Marrakech, Maroc)

Vincent Simonneaux ${ }^{1}$

Michel Lepage ${ }^{1}$

Dorian Helson ${ }^{1}$

Jérome Metral ${ }^{1}$

Sonia Thomas ${ }^{1}$

Benoît Duchemin ${ }^{1}$

Mohamed Cherkaoui ${ }^{2}$

Hakim Kharrou ${ }^{2}$

Brahim Beriami ${ }^{3}$

Abdelghani Chehbouni ${ }^{1}$

1 CESBIO

Unité mixte de recherche

(CNRS, UPS, CNES, IRD)

18, avenue Edouard-Belin

31401 Toulouse

France

<simonneaux@ird.fr>

<michel.lepage@cesbio.cnes.fr>

$<$ helsondo@voila.fr>

2 ORMVAH

Office régional de mise en valeur agricole

du Haouz

Marrakech

Maroc

<ikbalmohamed@hotmail.com>

<hakimkharrou1@yahoo.fr>

${ }^{3} \mathrm{ABHT}$

Agence de bassin hydrologique du Tensift

Marrakech

Maroc

<ab_ber@yahoo.fr>

Tirés à part : V. Simonneaux

Sécheresse vol. 20, $n^{\circ}$ 1, janvier-février-mars 2009

\section{Résumé}

Satellite monitoring of irrigation (SAMIR) est un logiciel de spatialisation de l'évapotranspiration (ET) et du bilan hydrique des cultures irriguées sur de grandes surfaces, basé sur l'utilisation d'images satellitaires. Cette source d'information fournit une vision synoptique et périodique de la localisation et du développement des cultures, donnée critique pour une estimation fiable de l'ET. Cette dernière est calculée au moyen de la méthode FAO, bien adaptée au calcul sur de grandes surfaces où I'information disponible sur le sol et les cultures est limitée. Le bilan hydrique de la culture est obtenu en couplant au modèle FAO un module sol en trois compartiments (surface, racinaire, profond). Le calcul du bilan nécessite des données liées au climat (estimation de l'ET de référence et précipitations), à l'occupation du sol et à la phénologie de la végétation (pour estimer les coefficients culturaux de la méthode $\mathrm{FAO}$, ces deux dernières données étant issues de la télédétection. Les données concernant l'irrigation sont soit introduites si elles sont connues, soit le plus souvent estimées à partir du calcul du bilan hydrique, en faisant des hypothèses sur la gestion de l'eau pratiquée et notamment sur le taux de stress hydrique toléré. Cet outil est développé en partenariat avec un utilisateur potentiel au Maroc, l'Office régional de mise en valeur agricole du Haouz (ORMVAH). L'irrigation estimée à l'échelle des secteurs irrigués peut être comparée aux apports connus des barrages et des seguias issues des montagnes pour estimer les pompages pratiqués dans la nappe souterraine. Dans un contexte de forte tension sur les ressources en eau, cette application montre les potentialités de l'imagerie satellitaire, pour le suivi du bilan hydrique des surfaces irriguées et la gestion des ressources à l'échelle du bassinversant.

Mots clés : bilan hydrique, évapotranspiration, irrigation, télédétection.

\section{Abstract}

Spatialized estimates of evapotranspiration of irrigated crops using remote sensing: Application to irrigation management in the Haouz plain (Marrakech, Morocco)

Satellite monitoring of irrigation (SAMIR) is software, which uses satellite images in computing spatialized estimates of evapotranspiration (ET) and irrigation water bud- 
gets for larges areas. Remote sensing offers a synoptic view of vegetation development, which is key information for reliable computing of ET. The ET is obtained using the FAO method, well suited for computation over larges areas where little information is usually available about crops and soils. The water budget is computed by linking to the FAO model a soil module including (1) a surface compartment accounting for soil evaporation, (2) a root compartment accounting for crop transpiration and (3) a deep compartment for water storage. The computation of the water budget requires climatic data (reference ET - namely "ETO" - and rainfall data), land cover data and crop development data (for estimating crop coefficients of the FAO method). These last two data types are obtained from remote sensing. Irrigation may be input when it is known, but it is usually estimated from the computation of the water budget, using hypotheses on water management modes and especially the average water stress level allowed. This tool was developed jointly with the "Office régional de mise en valeur agricole du Haouz" (ORMVAH) in charge of irrigation in the area. The irrigation estimates at the scale of irrigation sectors may be compared with known water inputs from dams and traditional seguias (channels deriving water from rivers) to estimate pumpings in the aquifer. In a context of strong pressure applied on water resources, we emphasize the potential of satellite images for monitoring irrigation and water management at the watershed scale.

Key words: evapotranspiration, irrigation, remote sensing, water budget.

\section{Introduction}

Les zones arides et semi-arides du bassin méditerranéen sont l'objet de tensions environnementales importantes, du fait de leur grande fragilité écologique et de la rareté des ressources en eau. La croissance et la transformation des besoins des populations accentuent la pression sur les ressources naturelles et peuvent engendrer des phénomènes de dégradation du milieu, souvent amplifiés par les changements climatiques.

Le projet SudMed, actuellement en cours à Marrakech (Maroc), développe des méthodologies intégrant des informations de terrain, des mesures satellitaires et des modèles physiques pour la gestion durable des ressources hydriques d'un bassinversant semi-aride, le Tensift [1]. L'accent est mis sur l'estimation de l'évapotranspiration (ET) de la végétation, variable clé du bilan hydrique des cultures, et donc de l'optimisation de l'irrigation.

\section{Site de l'étude}

Le bassin-versant du Tensift est constitué $d^{\prime}$ une vaste plaine semi-aride recevant environ $240 \mathrm{~mm}$ de précipitations annuellement, pour une ET potentielle de $1500 \mathrm{~mm}$. Cette plaine est adossée au Haut-Atlas, culminant au Toubkal à $4167 \mathrm{~m}$, qui reçoit environ $500 \mathrm{~mm}$ de précipitations, dont une partie sous forme de neige. Ce massif constitue ainsi le "château d'eau " du bassin du Tensift, en alimentant des cours d'eau permanents coulant vers la plaine, ainsi que la nappe localisée sous la plaine. L'ensemble du bassin est caractérisé par la rareté et la forte irrégularité spatiotemporelle des ressources en eau, contrainte à laquelle s'ajoutent, depuis quelques années, des périodes de sécheresse récurrentes. L'agriculture irriguée, concentrée dans la plaine du Haouz (environ 200000 ha), est de loin le plus gros consommateur d'eau de la région, puisqu'elle utilise environ $85 \%$ des ressources totales mobilisées. La gestion rationnelle de l'eau d'irrigation revêt donc une importance primordiale pour satisfaire les besoins en eau de tous les utilisateurs, et ce de manière durable.

\section{Pilotage de l'irrigation :}

les informations nécessaires

Les besoins en information pour un meilleur pilotage de l'irrigation dépendent de l'échelle de gestion considérée. Pour l'agriculteur cultivant quelques parcelles, l'information clé est la consommation des cultures au jour le jour, qui permet d'ajuster les apports d'eau, à condition d'en disposer à volonté. Pour estimer ces besoins, l'agriculteur peut en principe implémenter des méthodes simples, comme la méthode FAO (Food and Agriculture Organization) [2], à condition d'avoir accès à des données climatiques et de pouvoir quantifier la végétation présente dans ses champs. Cette estimation des besoins peut être encore améliorée si l'agriculteur dispose de mesures d'humidité du sol. Dans ce contexte local, le facteur limitant réside plus dans la maîtrise d'une technique d'estimation des besoins que dans les données nécessaires. Inversement, pour le gestionnaire régional de l'irrigation, I'information nécessaire est nettement plus importante et moins accessible. II s'agit, à l'échelle de secteurs d'irrigation, de savoir ce qui a été consommé depuis le début d'une saison, de connaître la consommation à un instant donné ou encore d'estimer ce qui sera nécessaire pour terminer la saison. L'eau agricole étant souvent issue de barrages de capacité d'approvisionnement limitée, ces informations sur le bilan hydrique permettraient d'optimiser la distribution pour répondre au mieux aux besoins. De plus, les eaux de surface étant le plus souvent insuffisantes, l'irrigation sollicite souvent les nappes phréatiques pour compléter les apports. Les bilans hydriques agricoles sont donc utiles aux agences de bassins pour estimer les prélèvements souterrains, permettre un meilleur contrôle des nappes et accéder à une modélisation hydrologique globale des bassins-versants. Pour répondre à ces besoins d'information à l'échelle régionale, le projet SudMed développe l'application informatique SAMIR (satellite monitoring of irrigation), outil d'aide à la gestion de l'irrigation à vocation opérationnelle fondé sur l'utilisation de séries d'images satellitaires. SAMIR permet de réaliser une estimation spatialisée de I'ET et du bilan hydrique des cultures à l'échelle des périmètres irrigués, et $d^{\prime}$ en déduire les apports en irrigation. Nous présentons dans cet article les grands principes de fonctionnement de cet outil.

\section{État de l'art}

Des outils existent déià, qui permettent un calcul du bilan hydrique d'un couvert végétal. Ainsi, GAPS [3] et BUDGET [4] utilisent des modèles de transfert sol-végétationatmosphère (TSVA) plus ou moins complexes à l'échelle de la parcelle. Plus simplement, le modèle CROPWAT [5] implémente la méthode $F A O$. Les deux premiers modèles nécessitent des données 
d'entrée trop complexes pour être spatialisés de manière opérationnelle. Inversement, CROPWAT présente un formalisme suffisamment simple pour être spatialisé, mais le développement réel de la végétation, variable critique qui conditionne le coefficient cultural, $n^{\prime}$ est en général pas connu en tout point, mais seulement estimé pour chaque culture.

Les applications offrant une réelle spatialisation de I'ET, prenant en compte notamment la spatialisation de la phénologie des couverts végétaux, sont nettement plus rares. Or, la télédétection spatiale offre maintenant une information spatialisée ef actualisée concernant la végétation. Les images satellitaires permettent, en effet, de distinguer différents types d'occupation du sol. Les séries temporelles d'images renseignent de plus sur le développement réel de la végétation, notamment par le biais d'indices de végétation comme le NDVI (Normalized Difference Vegetation Index). Cette information est bien corrélée à l'activité photosynthétique des végétaux, elle-même déterminante de I'ET. La disponibilité limitée et le coût des données haute résolution spatiale 110 $30 \mathrm{~m})$ et temporelle (10-20 jours effectifs) ont longtemps été un frein à ce type de valorisation, mais ils devraient rapidement devenir plus accessibles (Mission Formosat, Venus/GMES en projet).

Parmi les modèles utilisant les images satellitaires pour quantifier la végétation, le projet DEMETER développe un système d'aide à la conduite de l'irrigation basé sur le calcul de l'ET par la méthode FAO [6]. Cet outil utilise des séries temporelles d'images issues de différents capteurs (Terra ASTER, SPOT, Landsat TM, etc.) pour fournir périodiquement des coefficients culturaux destinés à être distribués aux agriculteurs. Cet outil ne calcule pas le bilan hydrique de la culture, qui nécessiterait la prise en compte de l'eau du sol. Des modèles plus complexes intègrent le bilan hydrique du sol. Zhang et Wegehenkel [7] proposent une approche basée sur la méthode FAO forcée par des images basse résolution MODIS et introduisent un modèle de sol empirique en trois réservoirs. La modélisation du bilan hydrique des cultures, ainsi réalisée, est englobée dans un modèle complet de bassin-versant, incluant fonte de neige ef débits à l'exutoire. Minacapilli et al. [8] proposent le système SIMODIS fondé sur une implémentation simple de la méthode FAO forcée par des images satellitaires, associé à un modèle physique de sol dont les paramètres hydrodynamiques sont déterminés par calibration sur des états hydriques observés.

L'information thermique fournie par certains capteurs (Landsat, ASTER, AVHRR) permet d'estimer un bilan énergétique de la surface et d'en déduire le flux d'ET instantané, par exemple grâce à la méthode SEBAL [9]. Ces méthodes ne permettent cependant pas, à elles seules, une intégration temporelle suffisante pour le contrôle du bilan hydrique de la végétation. II faut, pour cela, disposer entre deux observations d'une modélisation assez robuste pour interpoler l'ET. L'utilisation de modèles de culture permet $d$ 'atteindre ce but, mais est complexe à mettre en œuvre et nécessite des données détaillées inaccessibles sur de grandes surfaces. II est, en revanche, relativement aisé de se baser sur une modélisation type FAO pour faire cette interpolation, en quantifiant la végétation par des indices de type NDVI. Les estimations instantanées de flux, fourni périodiquement par la voie thermique, sont alors potentiellement très utiles pour contrôler le modèle FAO comme cela a été montré par Er-Raki et al. [10].

\section{Caractéristiques de SAMIR}

SAMIR fournit pour chaque pixel, au pas de temps journalier, des estimations spatialisées de l'ET et du bilan hydrique du couvert. L'originalité de l'approche est l'utilisation intensive de séries d'images satellitaires, afin de suivre au mieux le développement réel de la végétation au cours du temps, et non son développement supposé. Les images acquises sont également utilisées pour identifier ou affiner l'identification de l'occupation du sol au fur et à mesure de leur acquisition. Par rapport aux outils existants, les spécificités de SAMIR sont l'utilisation d'un formalisme suffisamment simple pour pouvoir être appliqué sur des zones étendues. L'utilisation de modèles de cultures pour simuler le développement de la végétation a donc été écartée dans la phase actuelle. L'ET des couverts végétaux est calculée selon la méthode FAO dual-crop coefficient, qui sépare la transpiration de l'évaporation du sol. Les coefficients culturaux nécessaires sont déterminés sur la base des NDVI issus des images satellitaires et interpolés entre les dates d'acquisition des images, linéairement ou par spline. On distingue différentes classes d'occupation du sol qui conditionnent la relation entre NDVI et coefficients culturaux. Le bilan hydrique du sol est réalisé grâce à la prise en compte d'un modèle de sol empirique en trois compartiments selon l'approche proposée par Zhang et Wegehenkel [7]. Bien que la méthode $F A O$ soit moins complexe que les méthodes basées sur les modèles physiques de type TSVA, sa sim- plicité et sa robustesse font qu'elle est bien adaptée à la spatialisation de l'ET sur de grandes surfaces, pour lesquelles les données exigées par les modèles plus complexes, concernant en particulier le sol et la végétation, ne peuvent de fait être obtenves. Cette méthode a été calée et validée, sur notre zone, pour les cultures dominantes : olivier, orangers et blé [11]. Son implémentation a été validée à partir d'une série satellitaire Landsat TM [12], ainsi qu'à partir de la désagrégation d'images basse résolution MODIS [13].

\section{Méthode de calcul du bilan hydrique}

L'ET d'un couvert est la somme de la transpiration de la végétation et de l'évaporation du sol. La méthode FAO modélise I'ET de n'importe quel type de végétation en la comparant, via des coefficients culturaux empiriques, à celle d'un gazon «standard » bien arrosé, placé dans les mêmes conditions de température, d'humidité de l'air, de vitesse du vent et de rayonnement solaire. Cette ET de référence (ETO) représente la "demande climatique» à un moment donné, elle peut être calculée au moyen de l'équation de Penman-Monteith [2]. À partir de là, l'ET d'un couvert quelconque, notée ETR (ET réelle), est obtenue par l'équation suivante :

$$
\mathrm{ETR}=\mathrm{ETO} \times(\mathrm{Kcb} \times \mathrm{Ks}+\mathrm{Ke})
$$

avec :

- Kcb coefficient basal crop déterminant la transpiration de la fraction de sol couverte par la végétation $(\mathrm{fc})$;

- Ks coefficient de stress hydrique ;

- Ke coefficient déterminant l'évaporation de la fraction de sol nu $(1-f c)$.

Le calcul de ETR nécessite donc trois types de données: des variables climatiques pour le calcul de l'ETO, l'occupation du sol et le développement de la végétation permettant d'estimer les coefficients culturaux Kcb et Ke. Le coefficient Ks est calculé à partir de l'état hydrique du sol et permet de réduire l'évaporation maximale du couvert en conditions bien arrosées, correspondant à $\mathrm{Kcb}$.

Le module sol de Zhang et Wegehenkel [7] comprend trois compartiments (figure 1). Le premier compartiment d'épaisseur fixe contrôle l'évaporation du sol, le deuxième est le compartiment racinaire qui contrôle la transpiration de la végétation, et dont l'épaisseur est variable dans le temps, car liée au taux de couverture de la végétation, lui-même contrôlé par le NDVI. Enfin, le compartiment profond complète le profil de sol jusqu'à une profondeur fixée à $2 \mathrm{~m}$. Ces trois compartiments se remplissent successivement lors d'une irrigation ou 


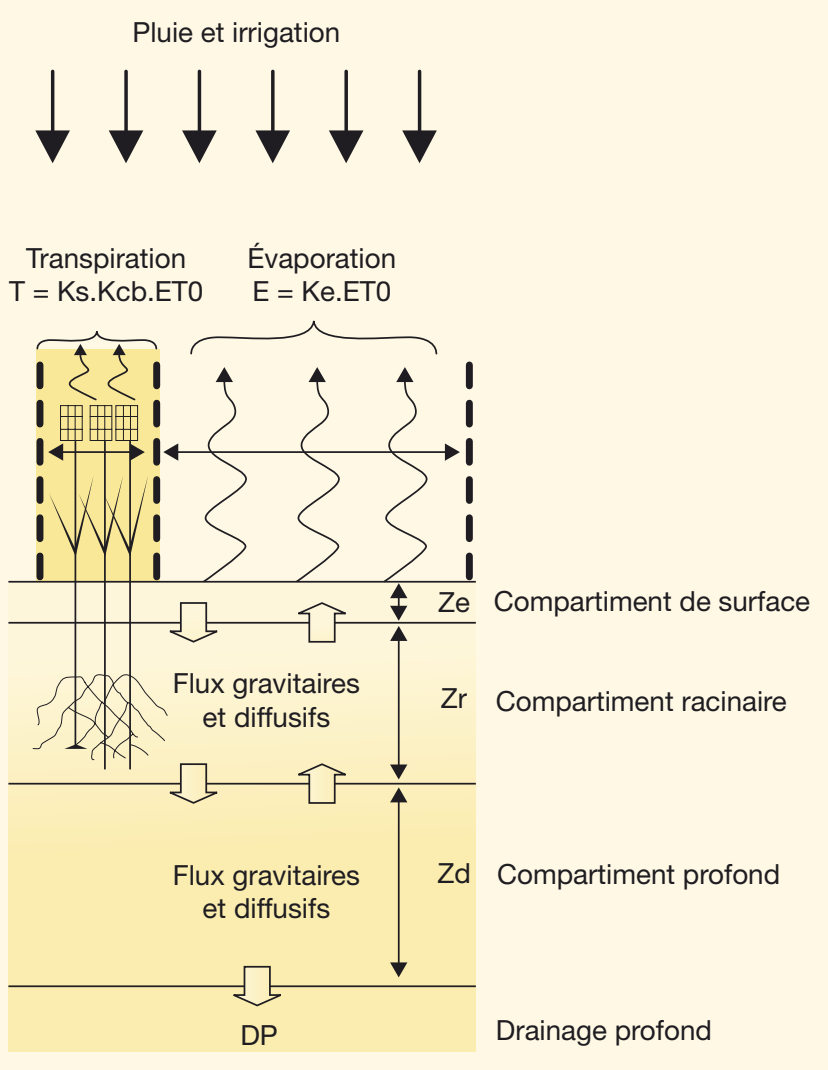

Figure 1. Modélisation des transferts d'eau entre sol, végétation et atmosphère dans SAMIR.

d'une pluie, le trop plein du troisième réservoir constituant le drainage profond.

Les teneurs en eau à la capacité au champ et au point de flétrissement sont définies par l'utilisateur, ainsi que les coefficients de diffusion permettant des échanges d'eau ascendants et descendants entre les compartiments en fonction de leurs différences de teneur en eau. Ce modèle de sol va permettre de calculer le coefficient $\mathrm{Ks}$ selon le remplissage du compartiment racinaire et, également, d'ajuster le coefficient $\mathrm{Ke}$ en fonction du taux de remplissage du compartiment de surface.

On considère, pour le compartiment racinaire, qu'une partie de la réserve utile est "facilement utilisable ", correspondant à un stress nul $(\mathrm{Ks}=1)$. Á partir de là, si le dessèchement se poursuit, le stress s'accentue linéairement jusqu'à vider la réserve utile $(K s=0)$. Un raisonnement analogue permet de définir le coefficient d'évaporation $\mathrm{Ke}$, constant et maximal, tant qu'on se situe dans une réserve "facilement évaporable » $(K e=1)$, et décroissant ensuite linéairement pour atteindre la valeur $\mathrm{Ke}=0$, lorsque le réservoir est vide.

À chaque itération, SAMIR calcule le bilan hydrique pour chaque pixel d'occupation

\section{Données en entrée et en sortie de SAMIR}

\section{- Module climat}

des résultats, cette méthode a l'avantage de fonctionner pour un nombre quelconque de stations d'entrée et de fournir des valeurs extrapolées réalistes qui ne sortent pas du domaine défini par les valeurs d'entrée, limitant ainsi les valeurs extrêmes que peut induire, par exemple, la méthode des splines. Enfin, SAMIR accepte des champs journaliers des variables climatiques générés par des modèles climatologiques, tels que ALADIN de la DMN (Direction de météorologie nationale marocaine), selon une grille régulière de $16 \mathrm{~km}$. Les pluies nécessaires au bilan hydrique sont introduites sous forme de données stationnelles qui seront alors interpolées spatialement.

- Module sol

Le module sol nécessite de définir des teneurs en eau à la capacité au champ et au point de flétrissement. Ces valeurs peuvent être considérées comme constantes sur la zone et définies par l'utilisateur. II est également possible d'introduire une carte pédologique comportant des unités de sol, pour lesquelles les teneurs en eau sont définies par horizon, SAMIR se chargeant alors de générer les propriétés moyennes des trois compartiments sol. À chaque modification de la profondeur racinaire, les propriétés des deux horizons inférieurs sont actualisées.

L'occupation du sol nécessaire à la spécification des relations NDVI-Kcb doit être fournie par l'utilisateur qui peut la réaliser au préalable par les méthodes de son choix. Dans un contexte pleinement opérationnel, si l'on se situe en cours de saison agricole, un problème de temporalité se pose pour ce type d'information. En effet, 'occupation du sol ne peut, en général, être cartographiée de manière fiable par télédétection qu'à partir du moment où une quantité suffisante $\mathrm{d}^{\prime}$ information est disponible, c'est-à-dire un certain nombre d'images, donc plutôt en fin de saison. Il est ainsi délicat $d^{\prime}$ utiliser SAMIR en début de saison, car des incertitudes existent quant à l'occupation du sol, qui ne se dissipent qu'au fur et à mesure de l'acquisition d'images.

Le module climat permet d'utiliser des statistiques des valeurs moyennes de l'ETO spatialisées au pas de temps journalier, publiées par la FAO (logiciel LocClim). II est également possible d'introduire des séries ponctuelles issues de stations de mesure, avec interpolation spatiale si plusieurs stations sont disponibles en utilisant la méthode de la pondération par la distance inverse. Malgré l'aspect souvent grossier
- Module phénologie

Le module phénologie offre la possibilité de fixer arbitrairement des profils type de $\mathrm{Kc}$ tels que ceux fournis par la méthode FAO. Toutefois, I'intérêt de SAMIR est d'utiliser des séries d'images satellitaires pour renseigner cette phénologie, typiquement une image tous les 15 jours, au cours de la saison végétative. Cette méthode a été utilisée par d'autres auteurs sur un nombre plus réduit $d^{\prime}$ images haute résolution [8, 14]. Différentes relations linéaires entre le NDVI et le Kcb existent ou peuvent être estimées à partir des données de la littéra- 
ture [14-16]. Elles peuvent être exprimées sous la forme suivante :

$$
\mathrm{Kcb}=\mathrm{A} \times(\mathrm{NDVI}-\mathrm{NDVImin})
$$

où NDVImin et $A$ dépendent de la végétation considérée.

Des irrigations distribuées spatialement peuvent être introduites au pas de temps journalier, SAMIR fournissant alors la dynamique hydrique du sol et de l'ET, et permettant d'identifier les éventuelles périodes de stress du couvert. Ce type d'utilisation pourrait convenir dans une optique de conseil au déclenchement de l'irrigation à la parcelle, mais ne correspond pas à l'objectif d'estimation de l'irrigation à l'usage des gestionnaires. De plus, cette utilisation en mode "déclenchement » nécessiterait de disposer d'informations nettement plus précises sur chaque parcelle pour fournir des informations pertinentes. L'utilisation normale de SAMIR est donc plutôt de déclencher automatiquement les irrigations, selon différentes contraintes définies par l'utilisateur concernant la fréquence et le volume des apports, ainsi que le niveau de stress accepté. On accède alors à une estimation globale de l'irrigation par zone, l'enjeu étant de paramétrer correctement le déclenchement de manière à reproduire le comportement «moyen » des agriculteurs. Il est alors évident que les irrigations proposées par SAMIR ne correspondront pas à la chronologie des irrigations réelles, ce qui n'a pas d'importance ici, puisque notre objectif n'est pas le conseil à la parcelle.

Le bilan hydrique est ainsi calculé pour chaque jour et pour chaque pixel. Cependant, compte tenu des incertitudes liées aux informations utilisées concernant en particulier le compartiment sol et les irrigations, les résultats doivent donc être considérés à des échelles spatiales ou temporelles supérieures.

\section{Application à la plaine du Haouz}

\section{ET de la saison 2002-2003}

L'ET a été estimée sur la plaine du Haouz, pour la saison 2002-2003, sur la base $\mathrm{d}^{\prime}$ une série de neuf images Landsat ${ }^{\mathrm{TM}}$. La zone irrigable couvre environ $2000 \mathrm{~km}^{2}$, occupée par $40 \%$ d'arboriculture, dont $80 \%$ d'oliviers, et $60 \%$ de céréales, dont $75 \%$ de blé. Les étendues emblavées et leur phénologie sont très variables d'une année à l'autre selon la pluviométrie du début de saison et la disponibilité de l'eau dans les barrages. On observe également une très forte variabilité spatiale selon les pratiques des agricul- teurs, liées à leur technicité et leur accès à l'eau. Dans ce contexte, les images satellitaires sont particulièrement utiles pour observer l'extension et le développement effectif des cultures annuelles.

Les images ont été corrigées radiométriquement en réflectance au sol pour calculer des indices NDVI. Une classification de l'occupation du sol a été réalisée à partir d'une analyse déterministe des caractéristiques des profils temporels de NDVI [12]. Les arbres étant majoritairement sempervirents, ils se distinguent par des valeurs de NDVI jamais inférieures à un seuil de végétation. Les annuelles correspondent aux pixels montrant une période de sol nu et une période de végétation. Enfin, les pixels de type arbre présentant en plus une forte variation de NDVI sont assimilés à des arbres associés à une sous-couche $d^{\prime}$ annuelles. On aboutit ainsi à une typologie en quatre classes, simple mais satisfaisante pour l'estimation du bilan hydrique à l'échelle des secteurs irrigués (sol nu, arbres, arbres avec sous-couche d'annuelles, annuelles). Du fait de la très forte hétérogénéité spectrale des classes due à la variabilité susmentionnée des itinéraires techniques et du développement de la végétation, on a montré que cette méthode de classification basée sur l'analyse des profils donne de meilleurs résultats que les méthodes classiques de type maximum de vraisemblance [12]. De plus, étant basée sur des critères à base physique, elle est relativement robuste et potentiellement applicable sans modification à $n^{\prime}$ importe quelle série $d^{\prime}$ images en réflectance sur la même zone.

Les coefficients culturaux ont été estimés à partir de relation NDVI-Kcb calibrée sur la zone d'étude $[11,16]$. Les ET ont été calculées en supposant un stress nul. Elles ont été validées ponctuellement avec succès sur trois parcelles de blé disposant de mesures d'ET réalisée par la méthode des flux turbulents (eddy correlation) (figure 2). On note une erreur moyenne journalière de $25 \%$, diminuant à $18 \%$ à l'échelle hebdomadaire. Une surestimation moyenne globale de $7 \%$ est observée pour l'ensemble de 160 jours de mesure disponibles pour les trois parcelles. Les valeurs sont localement affectées d'erreurs parfois importantes. Ainsi, la figure $2 A$ montre, en début avril, une période de stress hydrique pour laquelle I'ETR est inférieure à la valeur estimée, car SAMIR est réglé pour ne pas produire de stress fort. Inversement, la figure $2 B$ montre, en début de cycle, une période d'évaporation intense du sol liée à une irrigation réellement appliquée en début de cycle, qui n'est pas reproduite par le modèle. SAMIR manque en effet les irrigations appliquées, alors que la végétation n'est pas encore visible sur les images. Ce problème au démarrage des cultures annuelles souligne une lacune importante de l'outil, qui est l'impossibilité de reconnaître en temps réel l'occupation du sol en début de saison. Cette limitation est due au retard du signal NDVI par rapport à l'implantation de la culture. Ce retard est au moins partiellement rattrapé par la suite, lorsque la végétation est détectée et que se produit un rattrapage de l'irrigation pour assurer la croissance de la végétation enfin détectée. Malgré ces différences locales notables, les résultats montrent la relative robustesse du calcul d'ET par la méthode FAO sur le long terme. Bien que SAMIR n'utilise pas les irrigations réelles mais des irrigations estimées, les valeurs $d^{\prime} E T$ calculées sont très voisines des valeurs réelles, hors périodes particulières susmentionnées.

L'utilisation de Kcb issus de télédétection fournit ainsi une ET nettement plus proche de la réalité que l'ET calculée à partir des valeurs de la FAO relative aux conditions idéales de croissance (figure 3), et cela, d'autant plus que les cultures du Haouz souffrent souvent de divers stress (azote, eau, etc.) qui limitent leur développement [17].

\section{Estimation des irrigations et des pompages}

L'Office régional de mise en valeur agricole du Haouz (ORMVAH) connaît à l'échelle des secteurs les quantités d'eauirrigation délivrées en provenance des barrages, ainsi que les apports mesurés ou estimés à partir du réseau de seguias traditionnelles. Étant donné l'insuffisance chronique de la fourniture d'eau assurée par ces deux ressources, consécutive, notamment aux faibles remplissages des barrages constatés depuis plusieurs années, de nombreux exploitants ont massivement recours au pompage dans la nappe souterraine. Les quantités ainsi prélevées sont une variable clé pour les gestionnaires de la nappe, mais elles sont très difficiles à connaître, car elles résultent d'une somme de forages individuels. Sur la plaine du Haouz, des enquêtes exhaustives ont été réalisées, entre 2003 et 2006, par l'Agence de bassin hydraulique du Tensift (ABHT) pour recenser ces forages et quantifier l'eau extraite. Les évaluations ont été réalisées à partir des temps de pompages (méthode hydraulique), de 'énergie consommée (méthode énergétique) et des surfaces irriguées (méthode agronomique). La différence entre les valeurs d'irrigation totales estimées par SAMIR et celles mesurées par I'ORMVAH correspond à ces pompages et peut donc être comparée aux valeurs issues de 


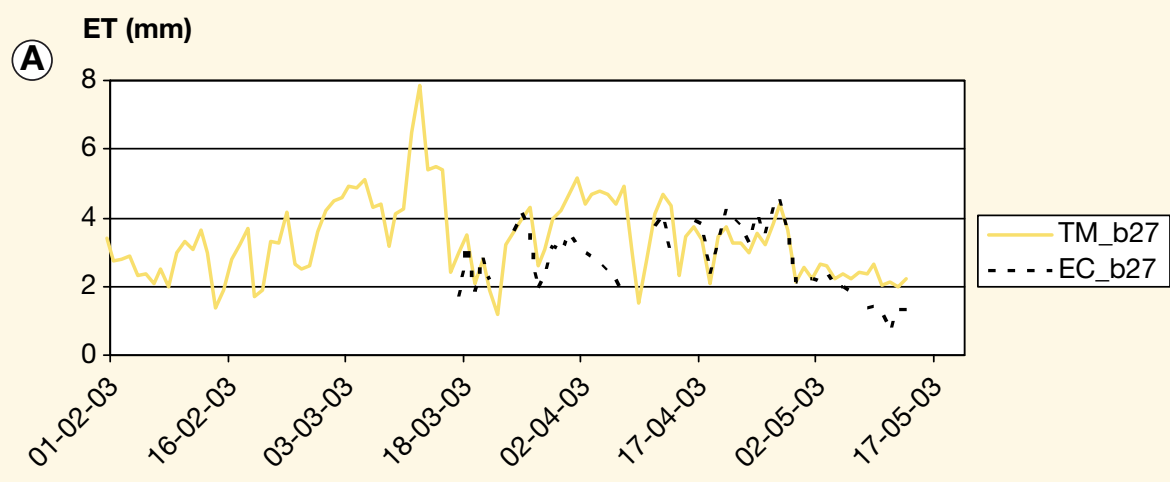

(B) $E T(m m)$

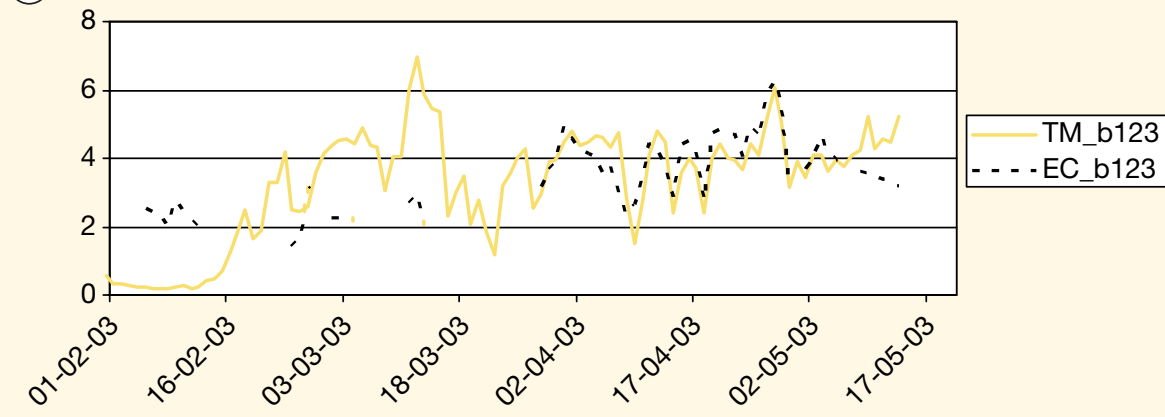

(C) ET (mm)

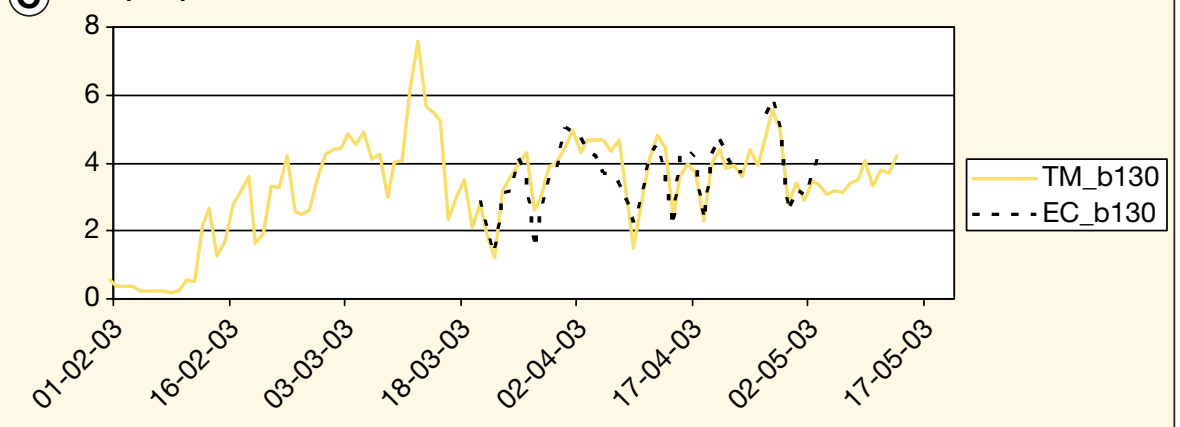

Figure 2. Comparaison entre l'évapotranspiration estimée par SAMIR (TM_*) et les valeurs mesurées au champ (EC_ ${ }^{*}$ ) pour trois parcelles (A, B et C) de la plaine du Haouz (Maroc).

l'enquête de l'ABHT. Une estimation des prélèvements dans la nappe a ainsi été réalisée pour l'année 2002-2003 et comparée aux valeurs d'enquête correspondant à la méthode agronomique (figure 4). On observe une relation significative, bien que peu déterminée, entre les lames d'eau pompées estimées et les valeurs enquêtées $\left(R^{2}=0,5\right)$. Les méthodes énergétique et hydraulique ne donnent aucun résultat significatif. La faiblesse de ces relations peut s'expliquer par la difficulté à réaliser des enquêtes de terrain fiables, les agriculteurs étant en général très peu enclins à déclarer leur usage réel par crainte d'être taxés sur leur consommation. Par ailleurs, la complexité du réseau de distribution de l'eau issue des barrages et des seguias fait que les évaluations des apports de surface par secteur sont probablement entachées $d^{\prime}$ erreurs. Enfin, les estimations d'ET issues tionnelle, la possibilité de prévision des besoins pour des échéances allant de la journée à la fin de saison est en cours de développement. Des prévisions de besoins en eau sont déjà réalisées par l'ORMVAH en début de saison sur la base des surfaces cultivées des années précédentes, en se basant sur des besoins en eau arbitraires des cultures (FAO). Elles sont ajustées deux fois au cours de la saison d'après les observations de mises en cultures réalisées.

Afin de mieux tenir compte du développement réel des cultures, nous développons actuellement un outil de prévision de la phénologie, basé sur les images acquises depuis le début de la saison. Cette prévision simple est basée sur une extrapolation graphique à partir des débuts de cycles observés. La qualité de la projection s'améliore donc avec l'augmentation du nombre d'images acquises, et on passe ainsi de simples scénarios en début de saison, à des prévisions plus fines au fur et à mesure de l'acquisition d'information satellitaire. II subsiste, dans tous les cas, une incertitude liée aux capacités de prévision des modèles météorologiques. Alors qu'il est possible de disposer de bonnes prévisions à échéance de quelques jours, celles-ci se dégradent nettement au-delà. Toutefois, dans le contexte d'une région semi-aride comme la plaine du Haouz, l'ETO à l'échelle $d$ 'une saison est relativement stable, et l'incertitude affectant le bilan hydrique concerne plus les précipitations.

Nous avons mentionné précédemment les possibilités offertes par l'information thermique à haute résolution spatiale $(60 \mathrm{~m})$ pour l'étude du bilan hydrique des surfaces. II existe également un potentiel de valorisation de l'information thermique satellitaire à haute répétitivité temporelle (journalière) et basse résolution spatiale (résolution 250 à $1000 \mathrm{~m}$ ) issue de satellites comme MODIS ou AVHRR, mais qui nécessite de mettre au point des méthodes de désagrégation spatiale spécifiques.

Par ailleurs, le satellite SMOS [18] fournira très prochainement des images microondes passives renseignant sur l'humidité des sols, même si la très basse résolution spatiale de ces images $(50 \mathrm{~km})$ nécessite également des méthodes de désagrégation spatiale pour son utilisation en agriculture irriguée.

Concernant l'occupation du sol, le problème d'identification en début de saison peut être partiellement résolu par la mise au point de méthodes d'actualisation progressive de ce type d'information. Ainsi, des cartographies d'années précédentes, utilisées comme hypothèses de départ en début de saison - éventuellement choisies selon des scénarios de disponibilités d'eau - pourraient être actualisées sur la base des images acquises au fil de la sai- 


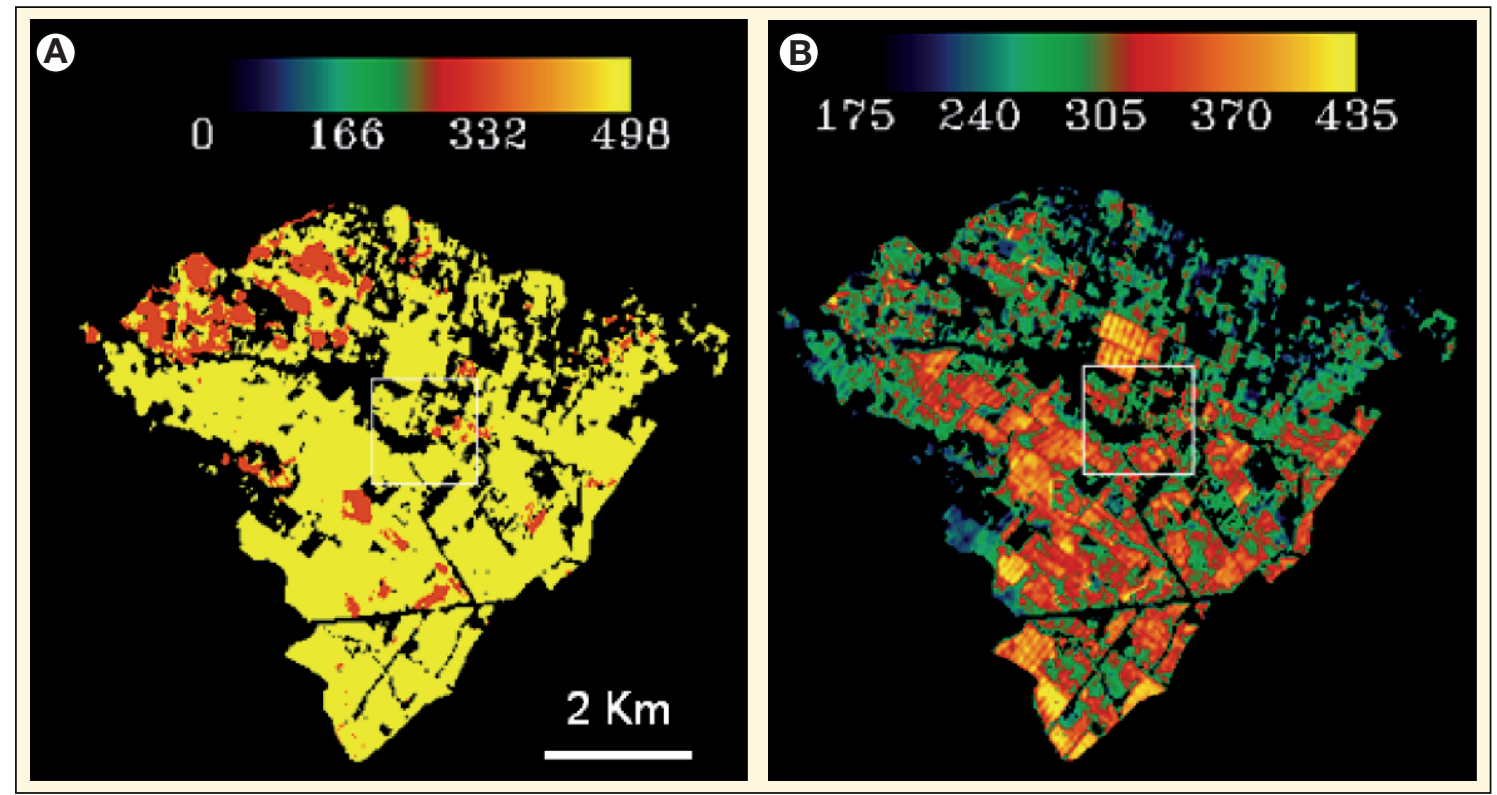

Figure 3. Exemple de l'intérêt du suivi satellitaire de la végétation sur le secteur irrigué R3 de la plaine du Haouz. A) Besoins théoriques estimés à partir des valeurs standard $\mathrm{FAO}: 13,1 \mathrm{Mm}^{3}$;B) Consommation réelle estimée par télédétection : 8,2 $\mathrm{Mm}^{3}$.

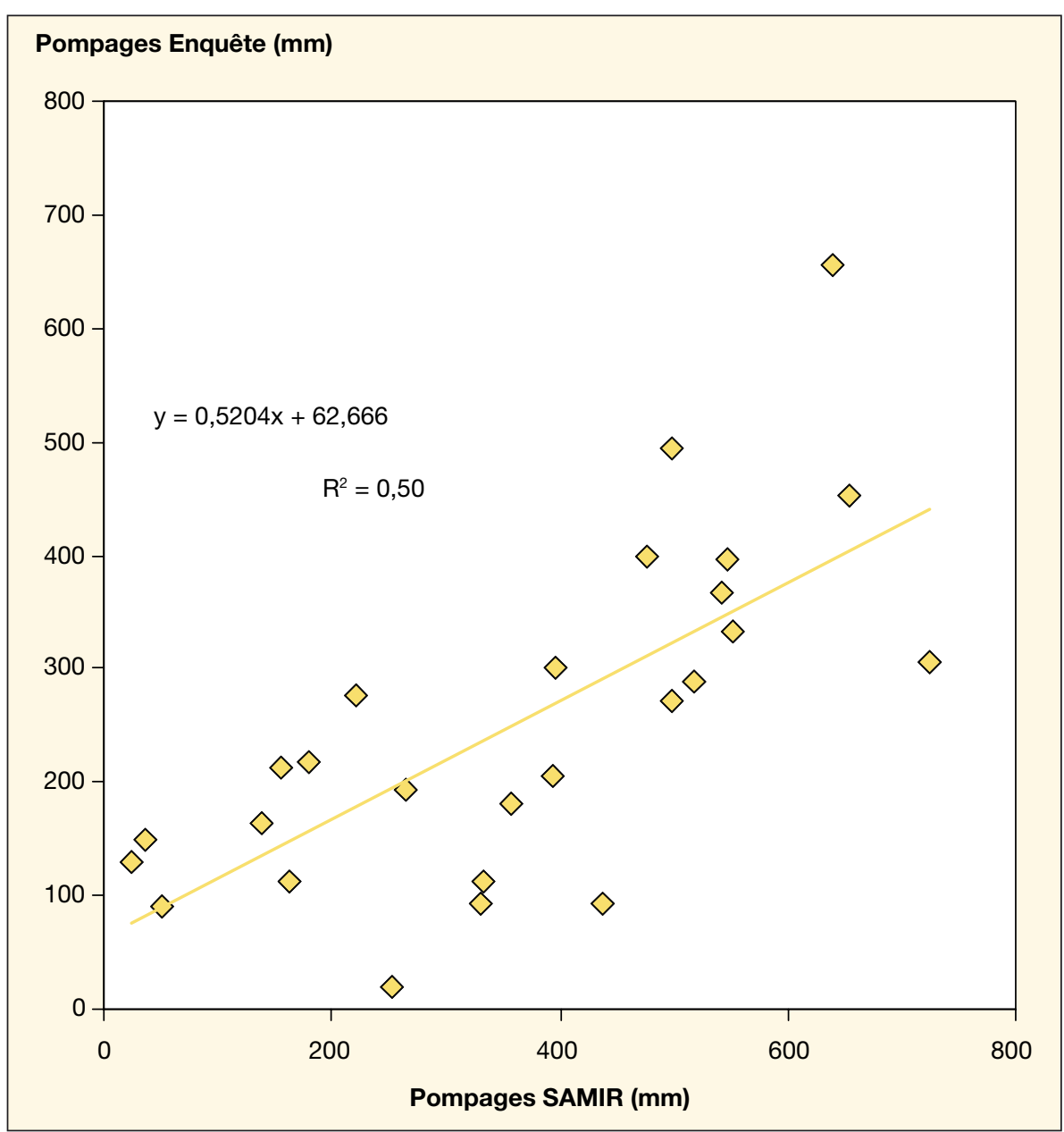

Figure 4. Comparaison entre les pompages issus de SAMIR et les valeurs issues de l'enquête ABHT 2003-2003 (méthode agronomique) pour les secteurs irrigués de la plaine du Haouz. son. D'autres types d'information satellitaire pourraient aussi permettre de détecter les cultures dès leur implantation et résoudre ainsi partiellement le problème du retard de détection lié au NDVI. Duchemin et al. [19] ont, en effet, montré que des images haute résolution spatiale $(8 \mathrm{~m})$ et très haute résolution temporelle (deux à trois jours) de type FORMOSAT font apparaître le travail du sol et les irrigations de présemis associés à la mise en place des annuelles par des variations importantes et rapides de la brillance du sol.

Enfin, des études ont montré la possibilité d'utiliser des images satellitaires basse résolution spatiale pour le suivi de la végétation et le calcul de son bilan hydrique [13]. Les méthodes proposées s'appuient sur la très haute répétitivité temporelle de ces capteurs (journalière) et sur des techniques de désagrégation de l'occupation du sol et des profils phénologiques associés.

\section{Conclusion}

Grâce à l'information qu'elle fournit périodiquement sur le développement des végétations cultivées, la télédétection est une source $d$ 'information particulièrement utile pour le suivi du bilan hydrique des surfaces irriguées. Sur cette base, SAMIR constitue une première version d'un système de suivi de l'irrigation fournissant des informations utiles pour la gestion de l'eau, particulièrement dans les régions où l'information sur l'irrigation est peu disponible. Toutefois, la validation des résultats néces- 
site d'importants jeux de données souvent difficiles à réunir. Un des défis les plus importants à relever pour ce type d'application est le développement des possibilités de prévision, nécessaire à son usage pleinement opérationnel, mais tributaire de nombreuses incertitudes sur les données d'entrée.

\section{Remerciements}

Ce travail a été réalisé dans le cadre du projet SudMed, réalisé conjointement par I'Institut de recherche pour le développement (IRD, France), la faculté des sciences de Semlalia de l'université Cadi-Ayyad de Marrakech (FSSM-UCAM), I'Office régional de mise en valeur agricole du Haouz (ORMVAH, organisme gestionnaire des périmètres irrigués de la zone) et l'Agence de bassin hydraulique du Tensift (ABHT). Nous remercions la Communauté européenne pour son soutien financier au programme PLEIADes, le CNES pour son soutien financier pour l'acquisition d'images satellitaires SPOT dans le cadre de l'initiative Isis du CNES (http://medias.obsmip.fr/isis), ainsi que le Comité mixte interuniversitaire francomarocain pour le financement du Programme d'action intégrée Volubilis MA/148/06. SAMIR est développé sous ENVI/IDL (@ RSI).

\section{Références}

1. Chehbouni A, Escadafal R, Duchemin B, et al. An integrated modeling and remote sensing approach for hydrological study in arid and semi-arid regions: the SudMed Program. Int J Remote Sens 2008 ; 29 : 5161-81.

2. Allen RG, Pereira LS, Raes D, Smith M. Crop evapotranspiration. Guidelines for computing crop water requirements. FAO Irrigation and Drainage Paper, no 56. Rome : FAO, 1998.
3. Buttler IW, Riha SJ. GAPS: a general purpose simulation model of the soil-plant-atmosphere system, Version 1.1 User's Manual. Ithaca (État de New York ): Cornell University Department of Agronomy, 1989.

4. Raes D, Van Goidsenhoven B, Goris K et al. BUDGET, a management tool for assessing salt accumulation in the root zone under irrigation. ICID 4th Inter-regional Conf on Envir-Water, 2730 Aug 2001, Fortaleza, Brazil.

5. Clarke D, Smith M, El-Askari K. New software for crop water requirements and irrigation scheduling. J Int Commission Irrigation Drainage 1998 ; 47 : 45-58.

6. Calera Belmonte A, Jochum A, Cuesta Garcia A, Montoro Rodríguez A, López Fuster P. Irrigation management from space: towards userfriendly products. Irrigation and Drainage Systems $2005 ; 19$ : 337-53.

7. Zhang $Y$, Wegehenkel $M$. Integration of MODIS data into a simple model for the spatial distributed simulation of soil water content and evapotranspiration. Remote Sens Environ 2006 ; 104 : 393-408.

8. Minacapilli M, lovino M, D'Urso G. A distributed agro-hydrological model for irrigation water demand assessment. Agric Water Manage 2008 ; 95 : 123-32.

9. Bastiaanssen WGM. SEBAL-based sensible and latent heat fluxes in the irrigated Gediz Basin, Turkey. J Hydrol 2000 ; 229 : 87-100.

10. Er-Raki S, Chehbouni A, Hoedjes J, Ezzahar J, Duchemin B, Jacob F. Improvement of FAO-56 method for olive orchards through sequential assimilation of thermal infrared-based estimates of ET. Agric Water Manage 2008 ; 95 : 309-21.

11. Er-Raki S, Chehbouni A, Guemouria N, Duchemin B, Ezzahar J, Hadria R. Combining FAO-56 model and ground-based remote sensing to estimate water consumptions of wheat crops in a semi-arid region. Agric Water Manage $2006 ; 87: 41-54$

12. Simonneaux V, Duchemin B, Helson D, ErRaki S, Olioso A, Chehbouni AG. The use of high-resolution image time series for crop classification and evapotranspiration estimate over an irrigated area in central Morocco. Int J Remote Sens 2007 ; 29 : 95-116.

13. Benhadi I, Duchemin B, Simonneaux V, Maisongrande $P$, Khabba S, Chehbouni AG. Automatic unmixing of MODIS multi-temporal data for inter-annual monitoring of land use at regional scale (Tensift, Morocco). Int J Remote Sens 2009 (sous presse).

14. Ray SS, Dadhwal VK. Estimation of crop evapotranspiration of irrigation command area using remote sensing and GIS. Agric Water Manage $2001 ; 49$ : 239-49.

15. Choudhury BJ, Ahmed NU, Idso SB, Reginato RJ, Daughtry CST. Relations between evaporation coefficients and vegetation indices studied by model simulations. Remote Sens Environ 1994; $50: 1-17$.

16. Duchemin B, Hadria R, Er-Raki S, et al. Monitoring wheat phenology and irrigation in Center of Morocco: on the use of relationship between evapotranspiration, crops coefficients, leaf area index and remotely sensed vegetation indices. Agric Water Manage 2006 ; 79 : 1-27.

17. Hadria R, Khabba S, Lahrouni A, Duchemin B, Chehbouni A, Carriou J. Calibration and validation of the STICS crop model for managing wheat irrigation in the semi-arid Marrakech-Al-Haouz Plain. Arabian J Sci Eng 2007 ; 32 : 87-101.

18. Kerr YH, Le Vine DM. Foreword to the special issue on the Soil Moisture and Ocean Salinity (SMOS) Mission. IEEE Transactions on Geoscience and Remote Sensing 2008 ; 46 : 583-6.

19. Duchemin B, Hagolle $O$, Mougenot B, et al. Agrometerological study of semi-arid areas: an experiment for analysing the potential of FORMOSAT-2 time series of images in the Marrakech plain. Int J Remote Sens 2008; 29: 5291-300. 